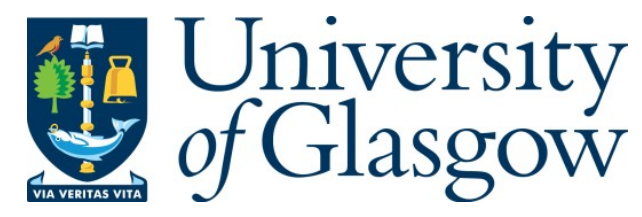

Phillips, D. B., Padgett, M. J ., Hannah, S., Ho, Y .-L. D., Carberry, D. M ., M iles, M. J ., and Simpson, S. H. (2014) Shape-induced force fields in optical trapping. Nature Photonics, 8 (5). pp. 400-405. ISSN 1749-4885.

Copyright @ 2014 Nature Publishing Group

http://eprints.gla.ac.uk/94910/

Deposited on: 08 July 2014

Enlighten - Research publications by members of the University of Glasgow http://eprints.gla.ac.uk 


\section{Shape-induced force fields in optical trapping}

D.B. Phillips ${ }^{1,2 *}$, M.J. Padgett ${ }^{2}$, S. Hanna ${ }^{1}$, Y.-L.D. Ho ${ }^{3}$,

D.M. Carberry ${ }^{1,4}$, M.J. Miles ${ }^{1} \&$ S.H. Simpson ${ }^{1}$

${ }^{1}$ H.H. Wills Physics Laboratory, University of Bristol, Tyndall Avenue, Bristol, BS8 1TL, U.K.

2 SUPA, Department of Physics and Astronomy, University of Glasgow, Glasgow, Scotland, U.K.

3 Department of Electrical and Electronic Engineering,

University of Bristol, Bristol, BS8 1UB, U.K.

4 School of Mathematics and Physics, The University

of Queensland, St Lucia, Brisbane 4072, Australia.

* Corresponding author. 
Abstract

Advances in optical tweezers, coupled with the proliferation of 2-photon polymerisation systems, mean that it is now becoming routine to fabricate and trap non-spherical particles. The shaping of both light beams and particles allows fine control over the flow of momentum from the optical to mechanical regimes. However, understanding and predicting the behaviour of such systems is highly complex in comparison with the traditional optically trapped microsphere. In this paper we present a conceptually new and simple approach, based on the nature of the optical force density. We illustrate the method through the design and fabrication of a shaped particle capable of acting as a passive force clamp; we demonstrate its use as an optically trapped probe for imaging surface topography. Further applications of the design rules highlighted here may lead to new sensors for probing bio-molecule mechanics, as well as to the development of optically actuated micro-machines.

It is well known that the high intensity gradients generated in a tightly focused laser beam can be used to trap and manipulate micron-sized particles [1]. An optically trapped sphere is an elegant example of a microscopic harmonic oscillator, capable of measuring fN-scale forces, which has proved invaluable for the study of molecular motors and single biopolymer mechanics [2]. However, there are other desirable features of a force field that can only be introduced by modifying the particle shape or dielectric structure beyond that of a simple homogeneous sphere [3, 4]. For example, optical torques can be applied to a particle whose symmetry has been lowered either by shape modification [5], or refractive index anisotropy. Control of orientation has most notably found applications in torsional experiments performed on biomolecules [6]. Other useful effects have been demonstrated, including anti-reflection coated beads for applying $\mathrm{nN}$ trapping forces [7] and optical wings that exhibit stable optical lift [8, 9]. More generally, shaping of colloidal particles allows control of their mechanical interaction with light at a fundamental level [10-12]; the recent proliferation of 2-photon polymerisation systems opens up new possibilities for designing novel optical tweezers experiments.

With increasing complexity of shape, the problem of predicting, and optimising, the 
force profile of the trapped particle becomes ever more challenging. Although specialised software packages are available to compute the optical forces [13], their use is not routine. In this paper, therefore, we address this problem and describe a straightforward method for predicting the optical forces acting on extended dielectric particles of general shape. We support the description with rigorous 3-dimensional T-matrix calculations. To illustrate the approach, we describe the design and fabrication of a passive force clamp based on a tapered cylinder and capable of applying a constant force over displacements of several microns. We demonstrate its use in an optically-controlled scanning probe microscope for ultra-low force imaging. Potential future applications of this device may include the imaging of sensitive biological membranes.

\section{Results}

\section{Background theory}

The trapping of spherical particles in the Rayleigh and Mie regimes is well understood [14]. In the more general case, the total optical force acting on an extended particle can be seen to derive from optical stresses distributed throughout the object. The precise form of the optical stress tensor remains contentious [15, 16], see Supplementary Information. In this work we favour the perspective of Brevik [17, 18], which is both straightforward to apply and consistent with the calculations and measurements described below. In particular, we calculate forces via surface integrals of the Minkowski stress-energy tensor, $\boldsymbol{T}_{\text {Mink }}$. The associated force density is then given by the divergence of $\boldsymbol{T}_{\text {Mink }}$, which differs from that obtained from $\boldsymbol{T}_{A b r}$ (the Abraham stress-energy tensor) by a small oscillatory factor whose cycle average is zero $[17,18]$.

The cycle-averaged optical stress, $\langle\mathbf{f}\rangle$, for homogeneous, non-absorbing, isotropic materials is confined to the material boundary and acts normally to it, pointing from the higher refractive index to the lower i.e. outward from a conventional optically trapped particle:

$$
\langle\mathbf{f}\rangle=\frac{1}{4}|E|^{2} \Delta \epsilon \delta(\mathbf{n}) \hat{\mathbf{n}}
$$

$E$ is the total electric field acting on the surface element, $\Delta \epsilon=\epsilon_{\text {particle }}-\epsilon_{\text {medium }}$ is the dielectric contrast, and $\hat{\mathbf{n}}$ represents the unit surface normal. $\delta(\mathbf{n})$ is a Dirac delta function, centred at the material surface. Equation 1 represents the optical force at a single point 
on the surface. The total optical force is given by integrating $\langle\mathbf{f}\rangle$ over the surface of the particle (i.e. summing all of the contributions over the surface) [19]. Clearly, the shape of the particle, as well as the distribution of intensity across its surface, will play a pivotal role in determining the optical force field.

We can gain an intuitive understanding of this situation by considering particles in the low refractive index contrast limit. In this case, the incident electric field is only weakly perturbed, and the total field ( $E$ in Eq. (1)) can be approximated, to zeroth order, by the incident field. The magnitude of the optical stress at each point on the surface is then given by the intensity of the illuminating light, and its direction by the surface normal. Such a description completely omits effects due to radiation pressure, polarization and geometric resonance but, nevertheless, provides a useful description of the contribution to the optical force derived from intensity gradients, which dominate in optical tweezers [4]. In reality, the objects considered here have moderate, rather than small, relative refractive indices. However they have low symmetry and do not exhibit sharp optical resonances so divergence from this approximation is smooth, and the above remains meaningful, as confirmed by the numerical simulations.

A simple illustration is given by a microsphere in a focused Gaussian beam. Figure 1a shows the characteristic linear restoring force as the sphere is translated laterally relative to the beam [20]. The curve has been calculated rigorously using T-matrix theory (see Methods). Insets (i) and (ii) show the distribution of optical stresses (black arrows) acting in a vertical plane through the centre of the model, evaluated using Eq. (1). The red arrow indicated the magnitude and direction of the total optical force on the particle (the surface integral of Eq. (1)). As the sphere is displaced further from the centre of the beam, the gradient of the illuminated part of the surface steepens, and the largest contribution to the optical stress progressively tilts over, increasing the lateral component of the optical restoring force. For large displacements the sphere leaves the high intensity part of the beam completely, causing a decrease in the magnitude of the optical stress and therefore a reduction in the total optical restoring force.

This description is readily extended to more complex particles. For example, Fig. 1b shows the behaviour of a cylinder positioned horizontally in a Gaussian trap. For small lateral displacements of the cylinder the restoring force is essentially zero, because most of the contributions to the optical stress are directed normally to the curved surface (see inset 
(i)). The lateral restoring force only becomes significant when the high intensity parts of the beam overlap one end of the cylinder (see inset ii), in which case the generated stresses act to move the cylinder back into the beam [21].

Figure 1c shows the effect of tapering the cylinder. The surface normals to the taper all possess an axial component, resulting in a constant lateral force that serves to draw the particle into the beam, even when the beam does not impinge on the end caps of the structure. The insets to Fig. 1c illustrate this behaviour. Providing the particle is only moderately diffracting, both displacements shown (insets (i) and (ii)) result in a similar lateral restoring force, as the orientation of the illuminated surface, and the optical power intercepting the particle, are the same in each case. The force plateau persists while the ends of the cone are outside the beam. It is evident from the above considerations that the qualitative force-displacement characteristics of any particle obeying the assumptions discussed above may be predicted from its shape.

\section{Experimental verification}

To verify the above predictions and demonstrate the robustness of the analysis, we fabricated particles with a range of tapers and characterised their behaviour. In a focused Gaussian beam an elongated object will naturally align its long axis with the beam axis, making Figs. 1b and 1c difficult to realize. However, horizontal trapping is possible using multiple beams [21] (Fig. 1d), and this was the approach used here.

Particles were fabricated using direct laser writing [22] (see Methods). An example is shown in Fig. 1e, consisting of two conical sections through which the object is trapped, and two spheres to provide tracking points for high-speed video stereo-microscopy [23]. The 3 -d coordinates of each sphere can be recovered to an accuracy of $\lesssim 5 \mathrm{~nm}$ at rates of up to $1 \mathrm{kHz}$, from which the position and orientation of the extended particle may be determined $[24]$.

Force-displacement curves were measured using a combination of Stokes drag and trap stepping techniques (see Methods). The Stokes drag method involves dragging the particle through the medium at different velocities, and observing its displacement from the trapping origin [20]. This technique cannot capture local minima in the force-displacement profiles, as the particle would simply jump past them. To circumvent this, the force characteristics 
were also measured by stepping the trap position and observing the velocity with which the particle returned to equilibrium [25].

Both of the above techniques rely on the fact that the effect of inertia is negligible at low Reynolds number, and so the optical $\left(\boldsymbol{F}^{\mathrm{opt}}\right)$ and viscous $\left(\boldsymbol{F}^{\text {hydro }}\right)$ forces equilibrate almost instantaneously. A stochastic force is present in both cases due to Brownian motion, but this is averaged out through repeated measurement. Therefore $\boldsymbol{F}^{\text {opt }}=\boldsymbol{F}^{\text {hydro }}=\boldsymbol{\xi} \boldsymbol{v}$ where $\boldsymbol{\xi}$ is the friction tensor, and $\boldsymbol{v}$ is the relative velocity of the particle and medium. $\boldsymbol{\xi}$ is calculated from the geometry of the particle and the viscosity of the fluid through which it moves (see Methods).

Figure 2 shows experimentally measured force-displacement curves for particles of four different tapers. Figure 2a shows the force-displacement curve of a cylindrical particle, measured using Stokes drag. As predicted in Fig. 1b, the force profile is symmetric with a shallow linear region (almost a plateau) for moderate displacements when the beams are far from the ends of the cylinders. Figure 2c shows the result of introducing a linear taper, measured using the trap stepping method, reproducing the form of the force curve predicted in Fig. 1c. We disregard the part of the force curve highlighted in red, which arises due to the switching time for the beam (see Methods). The equilibrium trapping point is now positioned towards the wider end of the cone, where the lateral force generated by the taper is balanced by that of the end cap. As the particle is displaced, a constant force plateau $(2.5 \pm 0.1 \mathrm{pN})$ extends for $\sim 3 \mu \mathrm{m}$ as the beam moves away from the larger end cap. Figure $2 \mathrm{~b}$ shows the results of T-matrix calculations indicating how the height of the force plateau is expected to vary with the degree of taper. As anticipated from Eq. (1), a steeper taper results in a higher force plateau. Lines corresponding to the experimentally measured cylinder and cone are highlighted.

In order to confirm that the restoring force is due to the local orientation of the particle surface, the force response of two curved tapers was also measured. In these cases the degree of taper varies along the length of the cone. Fig. $2 \mathrm{f}$ shows the curve for a convex taper. As this force curve is monotonic, both Stokes drag and trap stepping were employed, demonstrating their equivalence. Measurements for several step sizes of the trap stepping technique are plotted, once again allowing the portions of the curve resulting from the trap switching time to be identified and disregarded. The convex cone shape results in the beam sampling a steeper taper for larger displacements from equilibrium (see insets of simulated optical stress 
distributions), causing an increase in the restoring force and destroying the constant force plateau. A concave shape yields the opposite effect (Fig. 2d); the beam samples a shallower taper for larger displacements resulting in a reduction in the restoring force and a local minimum in the force curve. The restoring force increases again at larger displacements once the beam impinges on the end cap of the cone. Figure 2e shows T-matrix calculations for particles with a range of different curvatures. For the concave limit, $d r / d x=0$ when $r=r_{\min }$, while for the convex limit, $d r / d x=0$ when $r=r_{\max }$. The curvature is interpolated between these limits. Lines corresponding to the measured convex (black), concave (blue) and linear (grey) cases are highlighted. The forms of the experimentally measured force profiles agree well with both the anticipated behaviour from Eq. (1) and the rigorous Tmatrix calculations.

\section{A scanning probe application}

The degree of control over the force-displacement profiles of the optically trapped structures displayed above heralds the possibility of new applications. The shaped particles with linear tapers exhibit a force plateau extending over several microns of displacement (Fig. 2c). Here we demonstrate the use of such a particle as a scanning probe to image surface topography under conditions of constant force.

The probe is based on the design shown in Figs. 1d and 1e. We now also incorporate a tip mounted at the front and collinear with the probe, which is $3 \mu \mathrm{m}$ in length and tapers to a diameter of $200 \mathrm{~nm}$. Once pushed against the sample, the conical handles ensure that the tip maintains contact with the surface, while the maximum normal force exerted is capped at the value of the force plateau without the need for active feedback on the trap positions i.e. the probe acts as a passive force clamp. As the scanning probe is optically controlled, it has the additional advantage of exerting exceptionally low forces on a sample, compared to conventional scanning probe microscopy (SPM) [26-29]. Also, the sample is accessed from the side, unlike most SPM techniques which approach from above, allowing the topography of areas previously inaccessible to SPM to be investigated.

Figure 3 describes the imaging process. As there is no requirement for feedback, the probe is simply raster scanned along the side of the object. A single line-scan is achieved by updating the trap positions to translate the probe across the sample. Once a line-scan is 
completed, the sample is translated vertically relative to the probe by moving the objective lens with a piezo-electric focusing ring, and the process repeats. Within each line-scan, vertical motion of the probe is small (typically less than $200 \mathrm{~nm}$ ), so we do not need to apply any dynamic adjustment of the objective lens to maintain the probe at a constant height.

By tracking the positions of the two spheres on the probe, it is possible to calculate the coordinates of the probe tip throughout the scan. In this manner, and unlike other SPM techniques, Brownian motion provides a mechanism by which the tip explores the surface. The topography of the sample is reconstructed by finding the front surface of all loci visited by the tip, as shown in Figs. 3(e) and (g). In the images shown, the probe was scanned at $1 \mu \mathrm{m} / \mathrm{s}$, and each line-scan was separated by $200 \mathrm{~nm}$ in height.

The resolution of images produced by the passive force clamp is limited by three factors: the radius of curvature of the tip, how accurately the tip position can be tracked, and how fully the tip explores the surface. In the present case these factors result in a lateral resolution of $200 \mathrm{~nm}$ (due to the tip diameter, see Fig. 3c), and a depth resolution of $\sim 10 \mathrm{~nm}$. The depth resolution improves the longer the tip has time to explore a particular location, until system drift begins to degrade the measurement for larger timescales. How fully the tip explores the surface in a given time depends on the contact force, therefore, by maintaining a constant force throughout the image, the resolution is kept constant across the image. The length of the conical handles on the probe can be tailored to extend the force plateau to the depth over which surface corrugations in the sample are expected.

The use of an extended particle, with trapping and tracking points far removed from the interface under investigation, offers distinct advantages over a simple spherical probe. For example, it enables the position of an arbitrarily sharp tip to be accurately tracked, even when interacting with a highly diffracting interface. In addition, the trapping beams are unaffected by the presence of the sample, due to their spatial separation from it.

\section{Discussion}

In this article we have shown how an appreciation of the properties of the optical force density [Eq. (1)] can be used to obtain qualitative insights into the behaviour of shaped dielectric particles in optical fields. This understanding facilitates the design of optically trapped objects with specific force profiles for particular applications [30]. We have demon- 
strated this principle with the development of a passive force clamp, capable of exerting a constant force, of magnitude that can be tuned by the degree of taper, over a range of several microns. Force clamping is an important technique in single molecule bio-mechanical studies, conventionally relying either on feedback to update trap position (active force clamp) or the anharmonic region of the force response of an optically trapped sphere, with a range limited to $\sim 200 \mathrm{~nm}[31,32]$. The passive force clamp demonstrated here requires no feedback and can operate over a range defined by the length of the trapping cone. Here we have used it as a scanning probe to measure the surface topography of a sample, without the need to implement active feedback on the probe position.

The basic principles discussed above are quite general. The form of the force density is independent of indices of refraction, and structure and strength of illumination. For a particular incident field, modifying the shape of a particle has the effect of reorienting and redistributing the force density in an easily comprehensible fashion. As demonstrated, this leads to a simple and intuitive formulation for understanding, predicting and engineering the optical force field experienced by non-spherical particles. The approach can also be extended to inhomogeneous dielectrics: for objects possessing spatially varying permittivity, volume contributions directed along the permittivity gradient appear in the force density [17]. By controlling the internal distribution of the refractive index one could, therefore, modify the nature of the optical forces whilst leaving the shape unchanged. As nano-fabrication techniques advance, and the synthesis of objects of increasing complexity becomes possible [33], the approaches described here will further improve our ability to understand and utilize the mechanical properties of light.

\section{Methods}

Numerical calculations. Scattered fields are calculated using T-matrix theory which includes the effects of radiation pressure, polarization and optical resonance [34]. Incident, scattered and internal fields are expressed as discrete sums over sets of vector spherical wave functions (VSWFs). The optical scattering properties of the particle are expressed in its T-matrix. Since the particles are not necessarily convex, the T-matrix cannot be obtained using the embedded boundary condition method [35]. Instead we use the generalized multipole technique $[36,37$, applying it to each incident field basis function in turn, thereby 
establishing the T-matrix row by row.

The incident field is given by the localized approximation to a Gaussian beam [38], applying the translation theorem for VSWFs to generate coefficients for the two trapping beams. Forces are computed from integrals of the Maxwell stress tensor. The choice of stress tensor [15] and the question of whether a Lorentz force law, or similar, should be used instead [16], are matters of ongoing debate. However, we adhere to this approach noting that it is widely used elsewhere (e.g. [39]) and that there are sound theoretical reasons for assuming that the numerical difference between calculations performed with the Abraham and Minkowski versions of the stress tensor are negligible in the optical trapping regime [40]. Although the force density is a helpful device for understanding and predicting behavior, computation of the total optical force from the force density is not as straight forward as calculation from the stress tensor. However, we have compared the two approaches and found numerical agreement, as should be the case. The calculation based on the force density (Eq. (1)) requires careful treatment of the delta function, and evaluation of area elements on the surface of the object. A comprehensive account of these issues can be found elsewhere [19].

The friction tensors of the test particles, $\boldsymbol{\xi}$, were calculated using the bead models of Garcia de la Torre [41], in which the structure is modelled as a shell of hydrodynamically interacting spheres. In the present case, the friction tensor was deemed to have converged when each particle was represented by $\sim 10^{4}$ spheres.

Particle fabrication. Particles were fabricated from IPG photoresist in batches of 60 using a commercial 3D direct laser writing system (Nanoscribe Photonic Professional). They were harvested by being manually dislodged from the surface into a solution of deionised water and 0.5\% TWEEN 20 surfactant, using a wire mounted on a micro-manipulator, more details in [24] and [42]. The transfer efficiency using this method was 50-80\%.

Optical set-up. Optical trapping experiments were performed with a custom built holographic optical tweezers system equipped with a stereoscopic tracking system [23], a detailed description of the system can be found in [24]. The sample is imaged from two directions simultaneously. 2-D centre of symmetry tracking was performed on each image to find the centres of each tracking sphere. These were then combined using parallax to 
find the 3-D position of each tracking point, from which the position and orientation of the extended particle was determined.

Force-profile characterisation. Two techniques were used to measure the forcedisplacement characteristics of the optically trapped particles: Stokes drag [20] and trap stepping [25]. The Stokes drag measurements were performed by dragging the particle through the medium in a direction parallel to its long axis at a constant velocity, and measuring its displacement relative to its equilibrium position. This was repeated over a range of velocities. The particle was moved several tens of microns from the sample cell surface using the objective focus. A motorized $x-y$ translation stage (MS2000, ASI) was used to translate the sample at the required velocities. Each drag lasted for $4 \mathrm{~s}$, and the average axial displacement was calculated once the translation stage had attained a constant velocity.

The trap stepping technique involved stepping the traps in the direction parallel to the long axis of the particle, towards the tip of the cones, and observing the velocity as the particle returned to its equilibrium position. The steps in intensity are not instantaneous and the power in the new location takes several milliseconds to reach a maximum, resulting in a transient in the optical force. Comparing the force profiles for a variety of step sizes allows the transient to be identified and disregarded. As the equilibrium position for the tapered particle corresponds to the larger end being trapped, the method can only be used with steps towards the smaller end, where there is time for the transient to be disregarded before the particle returns to its equilibrium position. The orientation of the particle was monitored during characterisation, as any twisting would have altered the hydrodynamic drag force. No significant twisting $\left(\lesssim 3^{\circ}\right)$ was observed in any case.

\section{Acknowledgements}

This work is funded through an Engineering and Physical Science Research Council grant (United Kingdom). It was carried out with the support of the Bristol Centre for Nanoscience and Quantum Information, and using the computational facilities of the Advanced Computing Research Centre, University of Bristol - http://www.bris.ac.uk/acrc/. We are very grateful to Professor John Rarity for the use of the Nanoscribe Photonic Professional direct write laser lithography system for structure fabrication. M.J.M. and M.J.P. acknowledge 
Royal Society Wolfson Merit Awards.

\section{Author contributions}

D.B.P. fabricated structures, and designed and performed all experiments and analysis. S.H.S. formulated the concept of relating shape to optical force and performed all theoretical calculations, with support from S.H. Y.L-D.H. assisted with structure fabrication. M.J.P. provided support to the optical tweezers apparatus. M.J.M., M.J.P., and D.M.C. developed the concept of the scanning probe application. S.H.S., D.B.P. and S.H. wrote the paper, and all other authors provided editorial input.

\section{Competing financial interests}

The authors declare no competing financial interests.

\section{References}

[1] Ashkin, A., Dziedzic, J., Bjorkholm, J. \& Chu, S. Observation of a single-beam gradient force optical trap for dielectric particles. Opt. Lett. 11, 288-290 (1986).

[2] Block, S. M., Goldstein, L. S. \& Schnapp, B. J. Bead movement by single kinesin molecules studied with optical tweezers. Nature 348-352 (1990).

[3] Glückstad, J. Optical manipulation: Sculpting the object. Nature Photon. 5, 7-8 (2010).

[4] Simpson, S. \& Hanna, S. Stability analysis and thermal motion of optically trapped nanowires. Nanotechnology 23, 205502 (2012).

[5] Galajda, P. \& Ormos, P. Rotors produced and driven in laser tweezers with reversed direction of rotation. Appl. Phys. Lett. 80, 4653-4655 (2002).

[6] La Porta, A. \& Wang, M. D. Optical torque wrench: angular trapping, rotation, and torque detection of quartz microparticles. Phys. Rev. Lett. 92, 190801 (2004). 
[7] Jannasch, A., Demirörs, A. F., van Oostrum, P. D., van Blaaderen, A. \& Schäffer, E. Nanonewton optical force trap employing anti-reflection coated, high-refractive-index titania microspheres. Nature Photon. 6, 469-473 (2012).

[8] Swartzlander Jr, G. A., Peterson, T. J., Artusio-Glimpse, A. B. \& Raisanen, A. D. Stable optical lift. Nature Photon. 5, 48-51 (2010).

[9] Simpson, S. H., Hanna, S., Peterson, T. J. \& Swartzlander, G. A. Optical lift from dielectric semicylinders. Opt. Lett. 37, 4038-4040 (2012).

[10] Bishop, A. I., Nieminen, T. A., Heckenberg, N. R. \& Rubinsztein-Dunlop, H. Optical microrheology using rotating laser-trapped particles. Phys. Rev. Lett. 92, 198104 (2004).

[11] Trojek, J., Karasek, V. \& Zemanek, P. Extreme axial optical force in a standing wave achieved by optimized object shape. Opt. Express 17, 10472-10488 (2009).

[12] Palima, D. et al. Wave-guided optical waveguides. Opt. Express 20, 2004-2014 (2012).

[13] Nieminen, T. A. et al. Optical tweezers computational toolbox. J. Opt. A-Pure Appl. Op. 9, S196 (2007).

[14] Bowman, R. W. \& Padgett, M. J. Optical trapping and binding. Rep. Prog. Phys. 76, 026401 (2013).

[15] Pfeifer, R. N., Nieminen, T. A., Heckenberg, N. R. \& Rubinsztein-Dunlop, H. Colloquium: Momentum of an electromagnetic wave in dielectric media. Rev. Mod. Phys. 79, 1197 (2007).

[16] Mansuripur, M. \& Zakharian, A. R. Radiation pressure on a submerged absorptive partial reflector deduced from the doppler shift. Phys. Rev. A 86, 013841 (2012).

[17] Brevik, I. Experiments in phenomenological electrodynamics and the electromagnetic energymomentum tensor. Phys. Rep. 52, 133-201 (1979).

[18] Brevik, I. \& Ellingsen, S. Transverse radiation force in a tailored optical fiber. Phys. Rev. A 81, $011806(2010)$.

[19] Van Bladel, J. \& Van Bladel, J. Singular electromagnetic fields and sources (Clarendon Press Oxford, 1991).

[20] Simmons, R. M., Finer, J. T., Chu, S. \& Spudich, J. A. Quantitative measurements of force and displacement using an optical trap. Biophys. J. 70, 1813-1822 (1996).

[21] Phillips, D. et al. Optimizing the optical trapping stiffness of holographically trapped microrods using high-speed video tracking. J. Opt. 13, 044023 (2011).

[22] Cumpston, B. H. et al. Two-photon polymerization initiators for three-dimensional optical 
data storage and microfabrication. Nature 398, 51-54 (1999).

[23] Bowman, R., Gibson, G. \& Padgett, M. Particle tracking stereomicroscopy in optical tweezers: control of trap shape. Opt. Express 18, 11785-11790 (2010).

[24] Phillips, D. et al. An optically actuated surface scanning probe. Opt. Express 20, 29679-29693 (2012).

[25] Wang, F. et al. Resolving stable axial trapping points of nanowires in an optical tweezers using photoluminescence mapping. Nano Lett. 13, 1185-1191 (2013).

[26] Ghislain, L. P. \& Webb, W. W. Scanning-force microscope based on an optical trap. Opt. Lett. 18, 1678-1680 (1993).

[27] Friese, M. E. J., Truscott, A. G., Rubinsztein-Dunlop, H. \& Heckenberg, N. R. Threedimensional imaging with optical tweezers. Appl. Opt. 38, 6597-6603 (1999).

[28] Seitz, P. C., Stelzer, E. H. K. \& Rohrbach, A. Interferometric tracking of optically trapped probes behind structured surfaces: a phase correction method. Appl. Opt. 45, 7309-7315 (2006).

[29] Phillips, D. B. et al. Surface imaging using holographic optical tweezers. Nanotechnology 22, $285503(2011)$.

[30] Simpson, S., Phillips, D., Carberry, D. \& Hanna, S. Bespoke optical springs and passive force clamps from shaped dielectric particles. J. Quant. Spectrosc. Ra. (2012).

[31] Greenleaf, W. J., Woodside, M. T., Abbondanzieri, E. A. \& Block, S. M. Passive all-optical force clamp for high-resolution laser trapping. Phys. Rev. Lett. 95, 208102 (2005).

[32] Palima, D. et al. Optical forces through guided light deflections. Opt. Express 21, 581-593 (2013).

[33] Maragò, O. M., Jones, P. H., Gucciardi, P. G., Volpe, G. \& Ferrari, A. C. Optical trapping and manipulation of nanostructures. Nature nanotechnology 8, 807-819 (2013).

[34] Mishchenko, M. I., Hovenier, J. W. \& Travis, L. D. Light scattering by nonspherical particles: theory, measurements, and applications (Academic press, 1999).

[35] Kahnert, F. M. Numerical methods in electromagnetic scattering theory. J. Quant. Spectrosc. Ra. 79, 775-824 (2003).

[36] Hafner, C., Hafner, C. V., Hafner, C. V. \& Hafner, C. V. The generalized multipole technique for computational electromagnetics (Artech House Boston, 1990).

[37] Nieminen, T., Rubinsztein-Dunlop, H. \& Heckenberg, N. Calculation of the t-matrix: general 
considerations and application of the point-matching method. J. Quant. Spectrosc. Ra. 79, 1019-1029 (2003).

[38] Lock, J. A. \& Gouesbet, G. Rigorous justification of the localized approximation to the beamshape coefficients in generalized lorenz-mie theory. i. on-axis beams. J. Opt. Soc. Am. A 11, 2503-2515 (1994).

[39] Chen, J., Ng, J., Lin, Z. \& Chan, C. Optical pulling force. Nature Photon. 5, 531-534 (2011).

[40] Pfeifer, R. N., Nieminen, T. A., Heckenberg, N. R. \& Rubinsztein-Dunlop, H. Optical tweezers and paradoxes in electromagnetism. J. Opt. 13, 044017 (2011).

[41] Carrasco, B. \& García de la Torre, J. Hydrodynamic properties of rigid particles: comparison of different modeling and computational procedures. Biophys. J. 76, 3044-3057 (1999).

[42] Phillips, D. B. et al. Fashioning microscopic tools. Optical Trapping and Optical Micromanipulation X $\mathbf{8 8 1 0}$ (2013). 

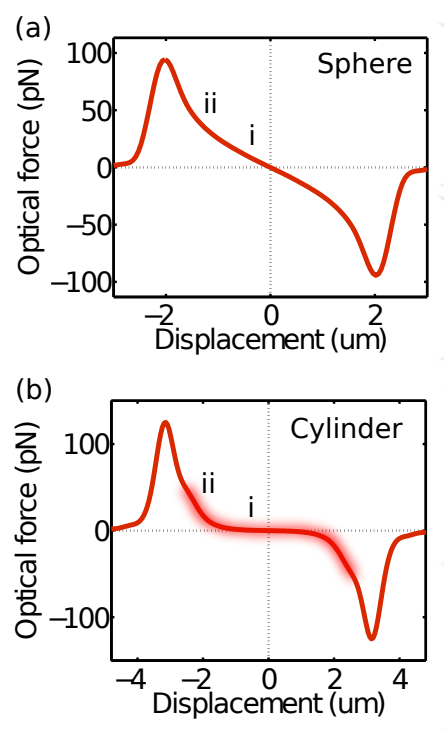

(c) Tapered Cylinder
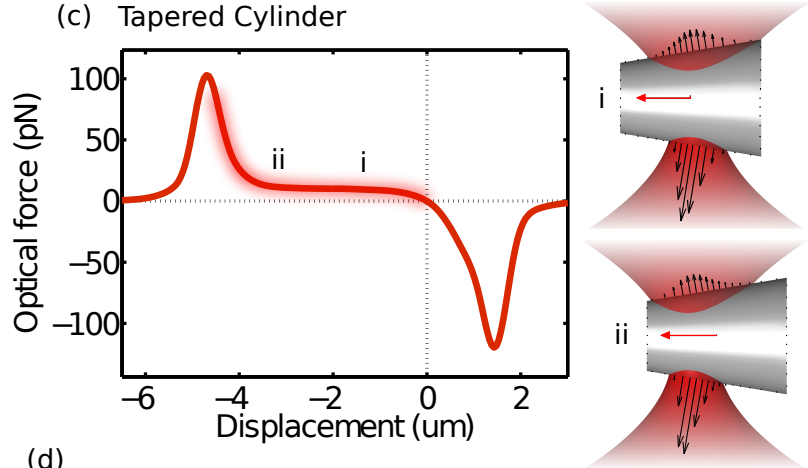

(d)

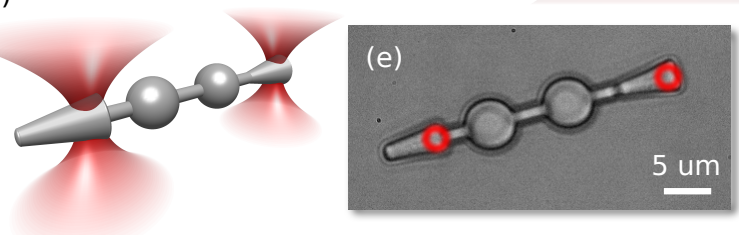

FIG. 1: T-matrix calculations showing the effect of particle shape on optical force

in a Gaussian beam. (a) Optical force exerted on a sphere (diameter $=4 \mu \mathrm{m}$ ) with lateral displacement from equilibrium. (b) The force-displacement profile for a horizontal cylinder (length $L=6 \mu \mathrm{m}$, radius $r=2.5 \mu \mathrm{m}$ ) as it is displaced laterally. (c) A similar plot for a tapered cylinder $\left(\mathrm{L}=6 \mu \mathrm{m}, r_{\max }=2.5 \mu \mathrm{m}, r_{\min }=1.5 \mu \mathrm{m}\right)$. Highlighted regions in (b) and (c) represent sections experimentally probed in Fig. 2. (d) A schematic model of a tapered test particle, shown at its equilibrium trapping position in a pair of traps. (e) An optical image of a particle similar to that in (d), fabricated using direct laser writing. Red circles indicate trap positions. All simulations are for a particle refractive index of 1.5 surrounded by water, and a laser wavelength of $1064 \mathrm{~nm}$. 

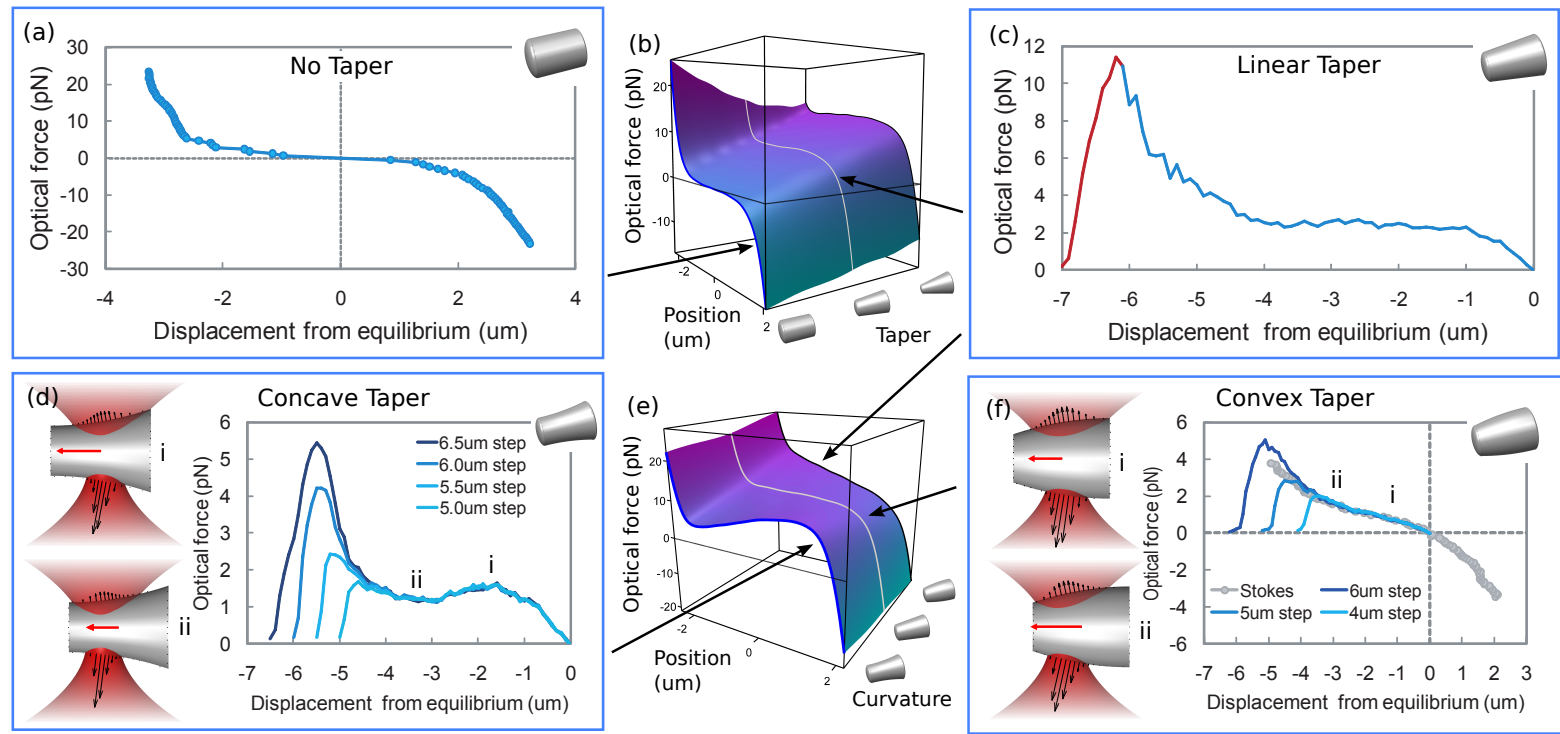

FIG. 2: Experimentally measured force-displacement profiles of horizontally trapped

cylinders and tapers. (a) The force-displacement profile of a cylinder (radius $r=2.5 \mu \mathrm{m}$, length $L=6 \mu \mathrm{m})$. (b) T-matrix calculations indicating how force-displacement profiles vary with taper ( $L=6 \mu \mathrm{m}, r_{\max }=2.5 \mu \mathrm{m}$, minimum radius $r_{\min }$ varies between 2.5 and $1 \mu \mathrm{m}$ ). (c) The profile of a particle with a linear taper $\left(\mathrm{L}=6 \mu \mathrm{m}, r_{\max }=2.5 \mu \mathrm{m}, r_{\min }=1.75 \mu \mathrm{m}\right)$. (d) The profile measured for a particle with a concave taper. (e) T-matrix calculations showing how the profiles vary with particle curvature. $r_{\max }=2.5 \mu \mathrm{m}, r_{\min }=1.75 \mu \mathrm{m}$ and $\mathrm{L}=6 \mu \mathrm{m}$ throughout. (f) The curve measured for a particle with a convex taper. In all cases the forms of the experimentally measured curves agree well with those predicted. 

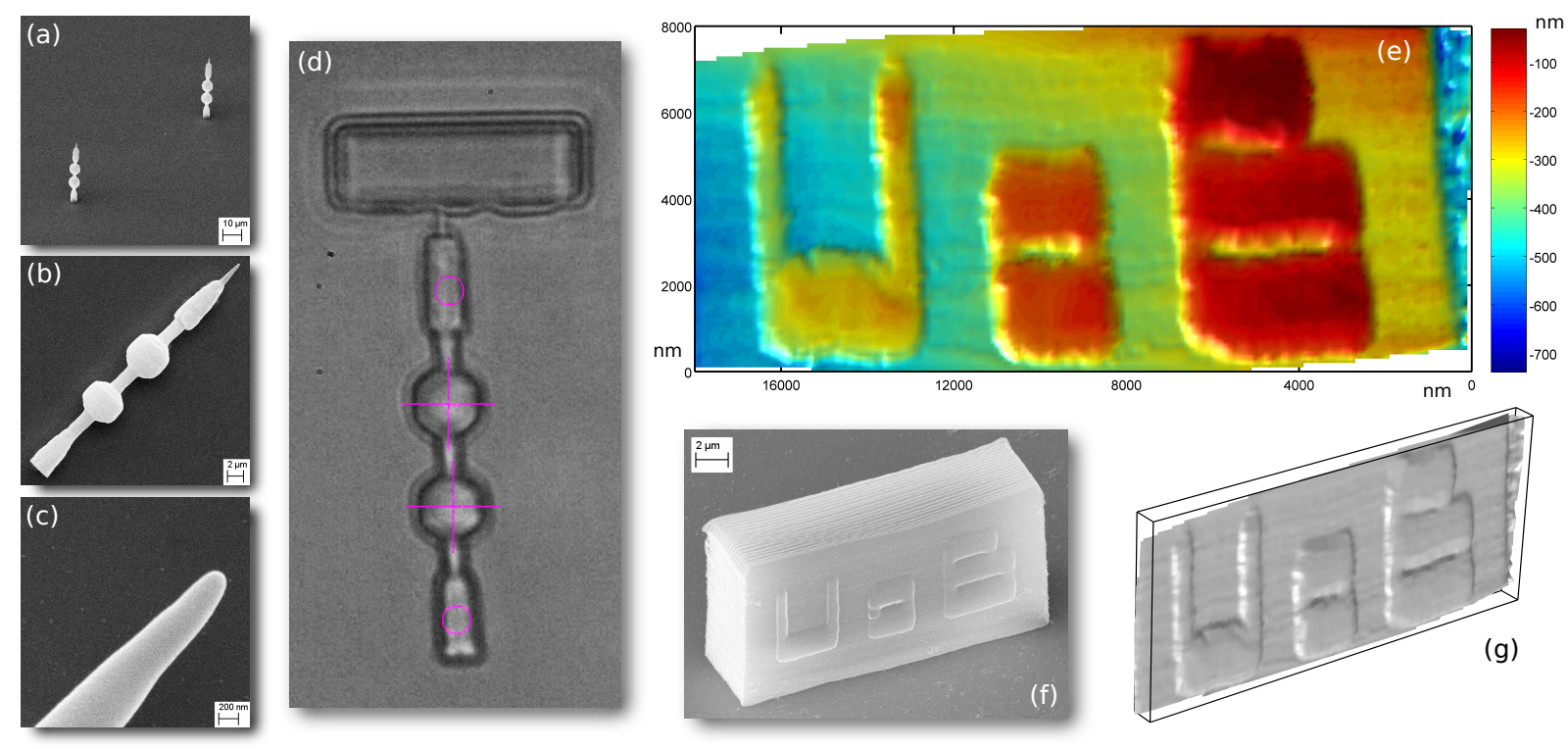

FIG. 3: Using a shaped particle as a scanning probe to image surface topography. (a) SEM image of two probes prior to harvesting. Vertical fabrication ensures the tip is as sharp as possible both axially and laterally. (b) SEM image of a probe dislodged from the substrate and lying on its side. (c) A close up SEM image of the $200 \mathrm{~nm}$ diameter tip of the probe in (b). (d) An optical image of a probe as it is scanned along the side of a calibration sample. Circles indicate the location of the optical traps. Crosses mark the tracked regions of the probe. (e) The surface topography of the side of the calibration sample recorded with the constant force probe. The height change across the image is $800 \mathrm{~nm}$. (f) SEM image of the calibration sample. (g) The same data as in (e), viewed as a $3 \mathrm{D}$ reconstruction for comparison with (f). 